\title{
The invisible college
}

\author{
Michelle Francl worries about the long-term effects of playing by pandemic rules.
}

On 12 March 2020 at 22:13 my classroom, and with it my students and colleagues, vanished. COVID-19 had driven my institution to remote instruction, rendering us an invisible college. Instead of gathering in a classroom that looks as though it could have been lifted from a set made for an Indiana Jones movie, lined with antique wooden drawers that slide open to reveal labelled boxes of minerals collected from around the world, my students were themselves dispersed across the globe, confined to small labelled boxes on my screen. We were suddenly everywhere and nowhere.

As I write this, it's nearly two months later and we are still an invisible college, tied together like Robert Boyle's unnamed virtuosi by common interests, not by location. By what measures I have, I have accomplished what I set out to do in January: guide and support my students along their path to becoming scientists. My worries have shifted from the immediate - can we make this move to remote modes of interaction and still be effective? - to what the long-term effects of playing under pandemic rules will be. What things are more visible now that we are invisible?

The first rule of the pandemic seems to be that there are no rules. Or rather that rules as they were set out at the start of the year would need to be remade. COVID-19 affects multiple organ systems and my syllabi were no exception. My neatly laid plans for midterm and final exams, office hours, problem sessions - and labs - were utterly disrupted. Whatever I was doing, it probably needed to be done differently. And my personal no-new-tech-in-the-middle-of-the-semester rule was going to be violated again and again as I added a webcam and microphone to my desk at home, learned two new collaborative platforms, experimented with three unfamiliar suites of video-editing software, and worked out what I would need to efficiently and effectively collect, grade and return some 2,500 pages of problem sets, worksheets and exams. And, like many of us, I had to manage it without losing momentum on other fronts: in my case, while continuing to prepare six classes a week, chairing the college's rank and tenure committee and, last but not least, running the chemistry department. Research? The bare minimum to keep it afloat was almost

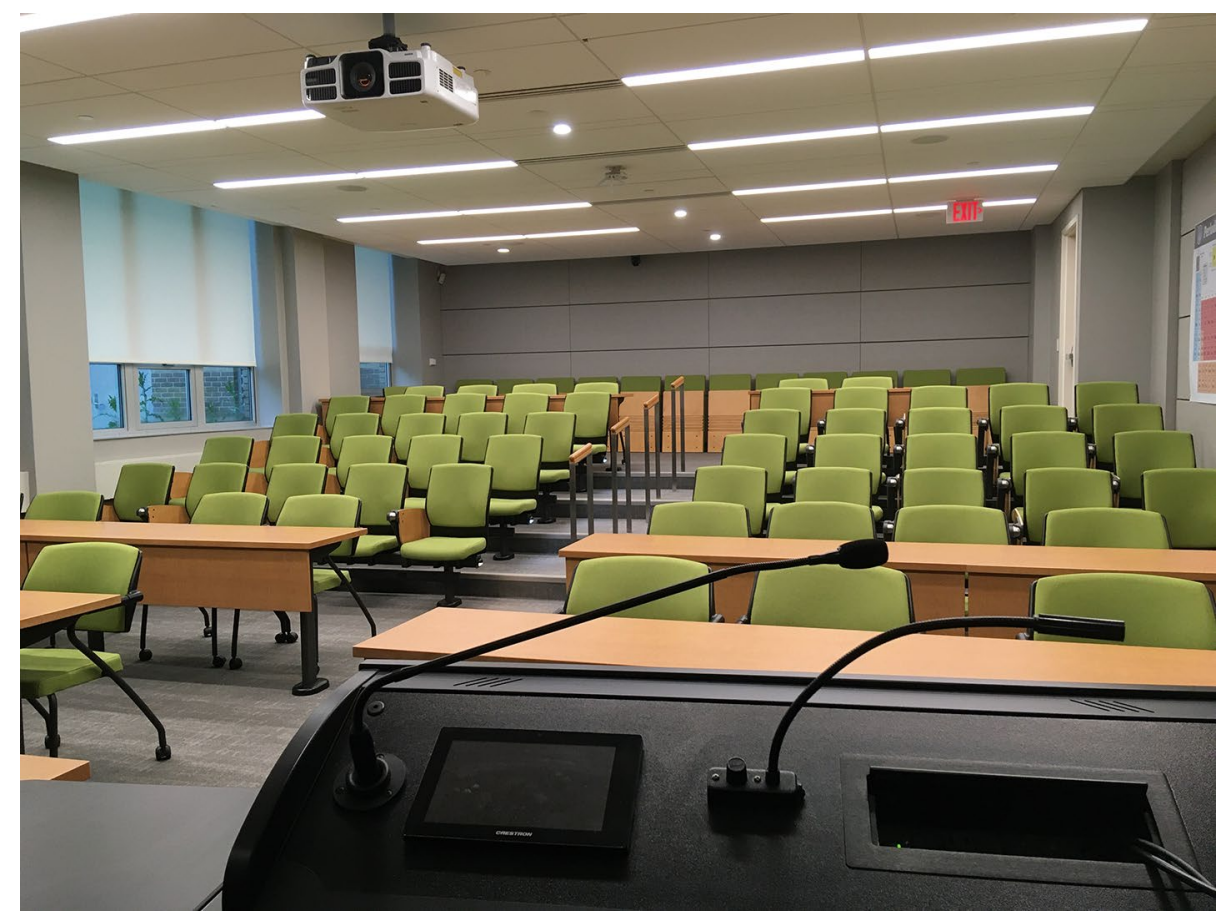

more than I could manage. Apologies to my collaborators.

My now defunct no-new-tech-mid-term rule was meant, I thought, to make me more effective as a teacher and researcher. It protected my undergraduate students' limited research time, enabling them to make progress unimpeded by changes in the software and hardware environment. And in class? No one wants to watch a lecturer fumble with an unfamiliar interface. Now, perforce, our students and colleagues are treated to our every misstep: "you're muted" or "you're not muted and your (pick one) dog is barking/phone is ringing/chipmunks

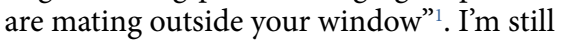
wincing at the statistical mechanics lecture where I clicked the wrong button, instantly and irrecoverably deleting the hour-long recording. (The bright side of that episode was that it didn't record the imprecation I uttered when I realized my error. I was, alas, not muted).

As much as I want the technological tools I wield to work seamlessly so that the science shines through, I recognize that the practice of science involves occasionally floundering until we figure out the right reaction conditions or the best method to get the energy of that interesting and highly strained structure to converge. The pandemic has made me once again realize the importance of letting our stumbling blocks and blunders in research and teaching be visible to students. When physical chemist and Nobelist Wilhelm Ostwald argued for making the bones of chemistry visible to students, he meant that students should be shown the underlying assumptions that chemists wield ${ }^{2}$. I might extend Ostwald's advice to include revealing our process as well, one that includes false starts, wrong turns and the uttering of an occasional obscenity.

Mercifully, I didn't have to do any of this backwards and in high heels: the second rule of the pandemic is no one sees what's on your feet. But my work life has delocalized into my home life, where my students and colleagues do see my overflowing bookshelves and occasionally catch the cat meowing at the second-floor window imploring me to let her in. In her essay 'Instructors, Please Wash Your Hair' Kristie Kiser argues that any sign of disorganization on my part, any whiff that I have unfolded laundry or small children in need of care hovering just out of sight of the camera is to be deplored ${ }^{3}$. In these uncertain moments, our students need to see us strong and 
unbowed without a hair out of place, she suggests. I get this, panic begets panic, my anxious students need no additional fuel. I suppose, too, it is good to show my students they are such a priority that I've changed out of my pyjamas for lectures or research meetings. But my students are certainly aware that I am at home, where there must be laundry to do and dishes to wash, seen or not. I fear such advice, however well-intentioned, threatens to subtly reinforce the narratives that scientists are to be wholly devoted to their science while someone else tends to dinner, the laundry and the kids, not only out of sight, but also out of mind. But scientists who are parenting cannot opt out of the quotidian drama.

It's not just flour and yeast that are in short supply as we bake out our pandemic anxieties. The final rule of the pandemic echoes the second law: you lose. The shift to working from home has meant not just the loss of our physical research spaces, but many scientists have experienced a shortage of space to think - the yeast that keeps science bubbling. As the coronavirus spread, there were many references to Isaac Newton's annus mirabilis. When Newton was sent home from Cambridge to isolate in the midst of an outbreak of the plague, he used that time to invent calculus and to come up with a universal theory of gravitation. What, many of these articles mused $^{4}$, will you do in the present time of isolation?

While, arguably, a new theory of gravitation is a high bar few of us will ever cross, pandemic or not, these articles also ignored the stark differences between Newton - a single 23-year-old isolated in his family's manor with (presumably) staff to cook and clean - and parents trying to work a full day while simultaneously looking after and teaching their small children. As paleobiologist Sam Giles wryly points out, Newton didn't have to deal with a three-year-old experimenting with entropy by tipping out and stirring together every bottle in the spice cabinet ${ }^{5,6}$. It's not just these one-off calamities that pull caregivers from their other work, it's the expanse of time available. Professor of engineering Vanessa Diaz's tweet ${ }^{7}$ nicely summarized the situation many scientists find themselves in; after spending 12 hours a day on childcare, and the evening doing routine work, 'when am I supposed to find the headspace and you know, TIME to write grants?!' Women often bear the bulk of household labour; single parents, now isolated at home, bear even more ${ }^{8}$. Editors have begun to notice a drop in journal submissions by women ${ }^{9}$, surely a proxy for the time lost by all caregivers.

The ability of some to use this time of isolation to think and to plan, to write up old work and propose new work is, in part, made possible by the efforts of those who kept everyone safe by staying home, making meals and taking care of the children. Science will suffer both from the immediate loss of the particular research that would have been done, but also from the loss of contributions from those whose careers are being impeded by slower publication rates and fewer grants impacts that will reverberate for many years to come. While it will cost us all, some will pay more than others ${ }^{8}$.

When my college returns to being gathered in one spot, I'm looking forward not only to seeing my students and colleagues again, but also hoping not to lose sight of what the pandemic brought into clearer focus. It has reminded me to showcase not just research's triumphs, but its travails as well; to reveal to my students the gritty reality of doing science. The pandemic has highlighted - and will undoubtedly magnify - inequities in the sciences, not just in the short term, when it comes to the number of papers submitted and grants written, but also in the subtle ways in which it will re-entrench stereotypes we have worked hard to diminish. What, I wonder, can I do - what will we all do - to make good these losses for our colleagues?

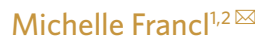

${ }^{1}$ Department of Chemistry at Bryn Mawr College, Bryn Mawr, PA, USA. ${ }^{2}$ Vatican Observatory, Vatican City, Vatican City State.

Twitter: @ichelleFrancl

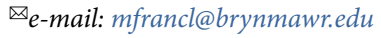

Published online: 17 June 2020

https://doi.org/10.1038/s41557-020-0497-1

References

1. Twitter https://twitter.com/BatesPhysio/status/1261345857183428 609 ? $s=20(2020)$.

2. Ostwald, W. The fundamental principles of chemistry: an introduction to all text-books of chemistry. vii-viii (Longmans, Green, and Co., 1909).

3. Kiser, K. Instructors, please wash your hair. Inside Higher Ed (16 April 2020).

4. Brockell, G. During a pandemic, Isaac Newton had to work from home, too. He used the time wisely. The Washington Post (12 March 2020).

5. Twitter https://twitter.com/GilesPalaeoLab/status/1258343470302 $998528 ? \mathrm{~s}=20(2020)$.

6. Twitter https://twitter.com/GilesPalaeoLab/status/1238033580363 845632 ? $\mathrm{s}=20(2020)$.

7. Twitter https://twitter.com/vdiazucl/status/1260833227759026176 ? $\mathrm{s}=20$ (2020)

8. Minello, A. The pandemic and the female academic. Nature (17 April 2020).

9. Viglione, G. Are women publishing less during the pandemic? Here's what the data say. Nature (20 May 2020). 